FOLIA SCANDINAVICA VOL. 24 POZNAŃ 2018 DOI: $10.2478 / \mathrm{fsp}-2018-0005$
Ssciendo

PRESSto.

\title{
ZWISCHEN RÄUMEN. (UN-)MÖGLICHKEITEN VON FREMDHEIT IN HENRI NATHANSENS ROMAN $A F$ HUGO DAVIDS LIV
}

\author{
CLEMENS RÄTHEL
}

Humboldt-Universität zu Berlin

ABSTRACT Henri Nathansen's highly successful bildungsroman Af Hugo Davids Liv, first published in 1917, tells the story of its Jewish protagonist from cradle to grave - with obstacles, adventures and challenges. But much more so, Nathansen offers a multi-layered narration of what it could mean to be a "noble" Jew. Providing a multitude of answers to that question, Af Hugo Davids Liv refuses any definition and thus opens a "third space" in which ambiguous and grained narrations of migration can take place, flourish and be understood in their own right. The article focuses on exploring these narrative interstices and spaces of in-betweenness and in doing so also (re-)discovers Nathansen's unique way of telling migration as socially always imminent.

\section{EINLEITUNG}

Im zweiten Band seines 1917 erschienen vierteiligen Bildungs- und Entwicklungsromans Af Hugo Davids Liv (AHDL) lässt der dänisch-jüdische Autor und Regisseur Henri Nathansen (1868-1944) den im Ringen mit sich selbst befindlichen Protagonisten einen Vorsatz fassen: „Ein Jude, edel, hilfreich und gut - ja, das wolle er sein!"“l (AHDL III: 100). Wie genau, lässt sich fragen, hat man sich so einen edlen Juden vorzustellen, oder genauer gesagt: Auf welche Art zeichnet Nathansen seine Ideen eines solchen? Noch dringlicher stellt sich diese Frage, wenn man sie vor dem Hintergrund des literarischen Vorbilds denkt, in welchem Goethe in seinem Gedicht Das

\footnotetext{
${ }^{1}$ Zitat im Original auf Deutsch.
} 
Göttliche dazu auffordert, als Mensch edel, hilfreich und gut zu sein. ${ }^{2}$ Das „,richtige“ Jüdischsein, so legt es Nathansens Formulierung nahe, berge eine spezifische Herausforderung, der sich der Roman zum Ende des Ersten Weltkrieges widmet.

Im Folgenden werde ich diese Darstellung genauer in den Blick nehmen und untersuchen, auf welche Weise sich Henri Nathansen in seinem voluminösen Erzählwerk Af Hugo Davids Liv den Herausforderungen sowie (Un-) Möglichkeiten eines edlen, hilfreichen und guten Juden widmet. Dabei verbindet er die Frage nach dem ,richtigen“ Jüdischsein mit Überlegungen, ob man gleichzeitig Däne und Jude sein könne, wie eine erfolgreiche Assimilation ausgestaltet sein müsse und wie fremd man im Eigenen bleiben dürfe. Auf welche Weise kartiert Nathansen nun das Spannungsverhältnis zwischen jüdischer Minderheit und dänischer Mehrheitsgesellschaft?

Dieser Komplex entwickelt eine besondere Brisanz vor dem Hintergrund der als ,großen Einwanderung“ (Blum, 1973: 37) rezipierten jüdischen Migration aus Osteuropa nach Dänemark zu Beginn des 20. Jahrhunderts. Die sich daraus ergebenen vielfältigen Reibungspunkte zwischen Neuangekommenen, der jüdischen Gemeinde vor Ort sowie der dänischen Mehrheitsgesellschaft legen bereits nahe, dass die jüdische Minorität weder als homogen noch durchgängig als nicht-dänisch bezeichnet werden kann. Stattdessen öffnen sich mehrdimensionale und wechselnd aufgeladene Räume, die zum einen neue (Erzähl-)Konzepte von Zugehörigkeit bieten und zum anderen verdeutlichen, wie das Perpetuieren von Gegensätzen zwischen dem vermeintlich Eigenen und dem Fremden dichotome Trennlinien erst erschafft und verfestigt.

Dass Migrationserzählungen und deren literarische Verhandlung vielstimmige Möglichkeiten offerieren, zeigt Nathansen in seinem Roman. In $A f$ Hugo Davids Liv geht er nicht offenkundig auf das als schwierig wahrgenommene Verhältnis der Neuangekommenen mit der Kopenhagener Bevölkerung ein, gleichzeitig lässt er jedoch eine ungemein vielstimmige Begegnung mit Fragen von Teilhabe und Identität(en) zu. Diese hybriden Kontakträume aufzuspüren, steht im Fokus dieses Beitrages.

\section{HENRI NATHANSEN}

Henri Nathansens literarisches Schaffen wird heute - wenn überhaupt vor allem mit seinem Aufklärungsdrama Indenfor Murene verknüpft, in dem auf der Bühne erstmals eine jüdisch-dänische Innensicht gezeigt wird und die titelgebende Mauer einer porösen Mitte weicht. (Räthel, 2018a) Das ungeheuer populäre Stück hat seit der Premiere 1912 allein am Königlichen Theater über

${ }^{2}$ Johann Wolfgang von Goethes Gedicht Das Göttliche beginnt mit dem Vers: „Edel sei der Mensch,/Hilfreich und gut!“ 
500 Aufführungen erfahren (Leicht \& Hallar, 1977), mehr als jedes andere Stück in diesem Zeitraum. ${ }^{3}$ Außerhalb Dänemarks dürfte Nathansen den wenigsten bekannt sein, dabei zählt er zu den produktivsten und erfolgreichsten Autoren der ersten Dekaden des 20. Jahrhunderts, der über die Landesgrenzen hinaus Erfolge verzeichnet. Neben einer bedeutenden Anzahl an Theaterstücken, von denen er etliche selbst am Königlichen Theater in Kopenhagen inszeniert, ${ }^{4}$ verfasst er Biografien und Theaterschriften, politische Pamphlete sowie zwei große Romane: Mendel Philipsen \& Søn - 1932 erschienen und 1992 von Liv Ullmann unter dem Titel Sofie verfilmt - und Af Hugo Davids Liv. Unter dem Eindruck der Machtergreifung der Nationalsozialisten im benachbarten Deutschland und später der Besetzung Dänemarks geht Nathansens literarische Produktion deutlich zurück. 1943 gelingt ihm die Flucht ins schwedische Lund, wo er sich 1944 unter dramatischen Umständen das Leben nimmt. (Svenson, 2016)

Etliche Werke seines umfangreichen Oeuvres - darunter seine beiden großen Romane - figurieren jüdische Protagonisten. Diese stellen eine Besonderheit dar. Nicht, weil es vorher keine jüdischen Charaktere gegeben hätte, ganz im Gegenteil: Sowohl die Dramatik als auch die Erzählliteratur haben eine große Anzahl jüdischer - oder zumindest mit deutlichen jüdischen Andeutungen versehener - Portraits hervorgebracht, die in den letzten Jahren auch in der literatur- und theaterwissenschaftlichen Forschung rezipiert worden sind, vorwiegend mit einem Schwerpunkt im 18. und 19. Jahrhundert. ${ }^{5}$ Neu ist jedoch, dass Nathansens Werke, allen voran Af Hugo Davids Liv, zu einer Zeit entstehen, in der die jüdische Migration aus Osteuropa nach Dänemark die Selbst- und Fremdwahrnehmung der etablierten jüdischen Minderheit in Dänemark verändert (Blüdnikow, Jørgensen, 1984: 304ff) und die Fragen nach den Voraussetzungen von Teilhabe und der Ausgestaltung von Assimilation erneut und vielfältig auf die Tagesordnung setzt.

${ }^{3} 2006$ wurde Nathansens Drama in den von der dänischen Regierung vorgelegten und heftig umstrittenen Kalturkanon aufgenommen, mit dem Hinweis auf die brennende Aktualität des Stoffes: ,[M]ed internationale jødeforfølgelser og holocaust har aktualiteten holdt sig op gennem 1900-tallet. Og holder stadig... Prøv for eksempel at skifte jødisk ud med muslimsk, så har vi balladen!“ (Kulturkanon 2006: 196)

${ }^{4}$ Mor har Ret inszeniert von Einar Christiansen (1905), Den gode Borger inszeniert von William Bloch (1907), Daniel Hertz inszeniert von Bloch und Nathansen (1908). Bei folgende Dramen führt der Autor auch Regie: Drømmen (1911), Indenfor murene (1912), Affceren (1913), Dr. Wahl (1915).

${ }^{5}$ Hingewiesen sei hier zumindest auf die zentralen Forschungsarbeiten von Jørgensen (1999), Müller-Wille (2013), Räthel $(2016,2018)$, Schnurbein $(2004,2007)$ und Schiedermair (2013). 


\section{3. „DIE GROßE EINWANDERUNG“}

Dänemark erlebt zu Beginn des 20. Jahrhunderts erstmals eine als Umwälzung wahrgenommene Migrationsbewegung (Blum, 1973: 37). Nach Pogromen in Russland verlassen seit 1903 viele Juden Osteuropa, die meisten emigrieren in die USA und nach Großbritannien. Etliche finden aber auch den Weg nach Dänemark, wobei der Großteil in Kopenhagen ansässig wird. Damit ändert sich zum einen die Struktur der jüdischen Gemeinde (Blum, 1973: 37), zum anderen modifizieren die Migranten das Bild „der Juden“ in der Öffentlichkeit: Die Einwanderer sprechen nicht Dänisch sondern Jiddisch, sie wohnen nachbarschaftlich in der Nähe der Synagoge, was dazu führt, dass der Abschnitt zwischen Rosenborg und Kongens Nytorv als ,jüdisches Ghetto“ bezeichnet wird (Blum, 1973: 38). Nicht nur die Differenz zur Mehrheitsgesellschaft wird als herausfordernd beschrieben, auch die Annäherung zwischen etablierten Juden und den Neuankömmlingen gestaltet sich schwierig: „Forend I kom, var vi danske af mosaisk tro, men nu betragter man os som jøder.“ (Borchsenius, 1969: 242) 1917 - im Erscheinungsjahr von Af Hugo Davids Liv - beendet ein königliches Dekret die weitere Einwanderung nach Dänemark.

Die eingangs vorgenommene Infragestellung der Gegensätze von Minderheit und Mehrheit wird vor diesem Hintergrund möglicherweise anschaulicher. Inmitten einer Periode von Veränderungen durch Zuwanderung liefert Nathansen in seinem Roman einen polyphonen Klang jüdischer aber auch nichtjüdischer Bilder. Es mag überraschen, dass Migration und Migrationserfahrung in Af Hugo Davids Liv keine unmittelbar ins Auge fallende Rolle spielen, Nathansen geht weder auf die Entwicklungen in Kopenhagen detailliert ein, noch erzählt er Migration als jüdische Urerfahrung. Was zunächst als Bildungs- und Theaterroman in der Tradition von Wilhelm Meister erscheint, generiert sich bei genauerer Betrachtung jedoch als eine von großer konzeptueller Offenheit geprägte Migrationserzählung.

\section{AF HUGO DAVIDS LIV}

Wie der Titel vermuten lässt, schildert der Roman Af Hugo Davids Liv in vier Teilen Lebensstationen seines jüdischen Protagonisten. Bereits die Untertitel der Bände verweisen auf zentrale Handlungsmotive: I. De Unge Aar, II. Byen, III. Flugten fra Festen, IV. Det Fjoerne Land. Aufgewachsen in der dänischen Provinz gelangt der junge Hugo David nach Kopenhagen, beginnt dort eine Ausbildung als Jurist bei seinem Onkel Isidor, bemerkt aber bald, dass seine große Liebe dem Theater gilt. Entgegen aller Einwände entscheidet er sich für eine Bühnenkarriere und etabliert sich bald als Schauspieler. Nach ersten Erfolgen lässt die Euphorie des Publikums nach und Hugo David wird mehr und mehr als unecht in seinem Spiel wahrgenommen. Die zunehmende 
professionelle Unzufriedenheit spiegelt sich auch im Privatleben wider: Die Liebe zu seiner Frau Ingeborg erkaltet zusehends, wirtschaftliche Schwierigkeiten stellen eine weitere Belastung dar, und als seine Frau ihn mit seinem Freund und schärfsten Rivalen am Theater hintergeht, bricht Hugos Welt zusammen. Nach einer langen Reise durch Europa kehrt er nach Kopenhagen zurück, er findet zu sich selbst und der nicht mehr für möglich gehaltene Erfolg auf der Bühne stellt sich ein. Versöhnt mit der Welt scheidet Hugo aus dem Leben.

Diese äußert knappe und stringente Zusammenfassung kann weder der verzweigten Handlung noch der besonderen Art des Schreibens Henri Nathansens gerecht werden. Der Eindruck, der Roman erzähle einen schwierigen, aber (literarisch) doch vorhersehbaren und schließlich erfolgreichen Lebensweg, trügt. Nathansen liefert in der Tat eher Vignetten, Beobachtungen, Blicke. Er erzählt eine große Anzahl von Begegnungen und schaut dabei auf und in seine Figuren. Die Augen des Lesers gleiten beständig über eine umfassende Personengalerie, die immer genauso viel über die Zeit und die Umstände erzählt wie über die jeweiligen Charaktere selbst. Af Hugo Davids Liv ist vielleicht am ehesten als epischer Roman zu lesen - episch dabei verstanden im Brecht'schen Sinn (Benjamin, 1989). Er lässt den Leser selten miterleben, was passiert, vielmehr bildet er ein Konglomerat aus Gedanken, Bild- und Personenbeschreibungen, Briefen, Düften und Klängen. Ein Kaleidoskop der Welten, durch die der Protagonist reist und stolpert, ein scharfsichtiger Blick in die dänische, später auch europäische Gesellschaft. Hugo fungiert dabei als großer literarischer Flaneur, an den sich die Beobachtungen und Vorgänge halten, die aber keineswegs seinen Lebensweg in einer Art fiktionalisierter Biographie nachzeichnen. Dabei gelingt es Nathansen - das sei vorweggenommen - keine Antworten auf die Frage zu liefern, was denn nun einen edlen, hilfreichen und guten Juden ausmache. Er postuliert keine Lösung, vermittelt in seinem Werk keine Ratschläge - weder an die Minderheit noch an die Mehrheit. Vielmehr zeichnet er eine Vielzahl von jüdischen und nicht-jüdischen Charakteren, mit unterschiedlichen Auffassungen darüber, welche Voraussetzungen und Bedingungen von Teilhabe zentral seien. Diese unterschiedlichen Positionen erzeugen Spannungsfelder, die Zwischenräume öffnen und sich einem EntwederOder entgegenstellen. Meine These lautet, dass Nathansens Roman in genau diesen Zwischenräumen - mit Homi Bhabha gesprochen „Dritten Räumen“ spielt und zugleich diese hybride Formation miterschafft.

\section{THIRD SPACE}

Homi Bhabha beleuchtet in seinem Werk The Location of Culture diese Art von Zwischenräumen und markiert sie als produktive Orte. Den „Dritten Raum" versteht er dabei nicht als Synthetisierung zwischen zwei bestehenden, 
festen Räumen, Polen und Positionen, vielmehr fordert er, diese „unreinen“ oder hybriden Ausgangslagen immer vorauszusetzen: „But for me the importance of hybridity is not to be able to trace two original moments from which the third emerges, rather hybridity to me is the ,third space' which enables other positions to emerge." (Rutherford, 1990: 211)

Bhabhas Konzept der Hybridisierung erscheint mir mit Blick auf Nathansens Roman besonders vielversprechend, weil es die Möglichkeit offeriert, Zwischenräume methodisch auszuloten und diese als „Schwellenräume zwischen den Identitätsbestimmungen“" zu fokussieren. (Bhabha, 2000: 5). Auch wenn Bhabhas Konzeptualisierung von Zwischenräumen vor allem mit Blick auf postkoloniale Lesarten Durchschlagskraft entwickelt, erweist sie sich auch in einem umfassenderen Verständnis von Migration und Migrationsliteratur als fruchtbar. So bezieht sich Søren Frank direkt auf Bhabha, wenn er gegen ein verengtes Verständnis von Migrationsliteratur argumentiert, das traditionell darauf ziele, die physisch-geographischen Bewegungen des Autors oder der Protagonisten von einem Ort zu einem anderen Ort als kennzeichnend wahrzunehmen. (Frank, 2012: 6) Damit, so Frank, verfestigten sich mononationale Rahmungen und Kategorisierungsprozesse. (Frank, 2012: 4) Im Gegenteil plädiert er dafür, Migration nicht nur als räumliche Bewegung im herkömmlichen Sinn zu betrachten, sondern auf zeitliche und mentale Prozesse auszudehnen - und damit dynamische textuelle Strategien zu beleuchten. Hier schließt er direkt an Bhabha an, wenn er den Mehrwert in der Auseinandersetzung mit so genannten Migrationserzählungen an deren performativen Strategien misst, die „,entrifugaliserer de personlige, kulturelle og nationale identiteter såvel som at bringe de interne forskelle frem i forgrunden med henblick på at kontrastere de monokulturelle billeder af nationen og dets befolkning med kosmopolitiske, mudrede billeder.“ (Frank, 2012: 7) Wie genau aber erschafft Nathansen diese kosmopolitischen Bilder? Was erzählen sie?

\section{ZWISCHENPOSITIONEN}

Folgt man Hugo David durch sein literarisches Leben, fällt auf, dass für Nathansen die Zwischenpositionierung des Protagonisten als Leitmotiv fungiert. Hugo David ist beständig hin- und hergerissen: unter anderem zwischen Land und Stadt, dem Theater und der „Wirklichkeit“, der Liebe zu Ingeborg und zu Karl, seinem Heim- und Fernweh sowie dem Bestreben, als Jude sowohl unsichtbar als auch besonders sein zu wollen oder zu müssen. Immer wieder stellt sich der Protagonist die Frage nach den (Un-)Möglichkeiten von Zugehörigkeit: „Han syntes, at hans Liv fra den tidligste Barndom havde varit en ensom Vej - Vejen førte stadig frem, men aldrig hjem.“ (AHDL III: 35)

Man kann dies wie Tine Bach in ihrer Untersuchung Exodus als typisch jüdische Heimatlosigkeit interpretieren (Bach, 2004), die für das Leben in der 
Diaspora charakteristisch ist. Die Sehnsucht nach Zugehörigkeit wird in dieser Lesart mit der Aussicht auf deren Unmöglichkeit beantwortet. Sobald Hugo begreift, dass er Jude sei, so Tine Bach, werde er heimatlos. Seine Ehe mit einer Nicht-Jüdin sowie die Entscheidung für eine Bühnenkarriere versteht sie dann auch als Aufruhr gegen jüdische Traditionen und sein vermeintliches Scheitern an beidem als logische Konsequenz dieser ,illusorischen“ Bemühungen. (Bach, 2004: 237) Bachs Essentialisierung der jüdischen Erfahrung die sie im Fall von Af Hugo Davids Liv mit Verweisen auf den vermeintlich autobiographischen Charakter des Romans zu unterstreichen versucht (Bach, 2004: 238f) - verkennt jedoch die Spannungsfelder und Mehrdeutigkeiten des Textes.

Ja, es gibt bei Nathansen diese „Hjemløshed og Tristhed“ im Blick (AFHDL III: 75) verbunden mit der Unsicherheit über das (auch geographisch) Eigene: „Var dét Forbandelsen - aldrig at kunne samles under noget fælles Tag, aldrig at kunne samles i et fælles Fædreland, fordi Nærheden vilde splitte dem og kun Afstanden kunde forene dem?" (AHDL II: 100) Die Frage nach Heimat und Zugehörigkeit kann dabei scheinbar zu keinem Zeitpunkt beantwortet werden und wenn Hugos Onkel vorausdeutend aus Karl Gutzkows Drama Uriel Acosta zitiert, klingt an, dass Hugo selbst im Tod nicht wissen könne, wohin er gehöre: „Nirgends find' ich ein Grab, bei Christen nicht, nicht Juden - ich bin von denen die am Wege sterben. ${ }^{\text {"6 }}$ (AFHDL II: 171) Schaut man aber den Text genauer an, wird deutlich, dass Nathansen vielfältig auf Zwischenräume rekurriert bzw. diesen einen zentralen Platz einräumt: Sicher mag die Vorstellung, am Wege zu sterben, zunächst wenig verlockend daherkommen, jedoch deutet sie bereits an, dass es eine Möglichkeit jenseits eines Entweder-Oder geben kann. Und diese hybriden Möglichkeiten erzählt Nathansen sehr umfangreich, der gesamte Roman liefert - auf vielen Ebenen eine multiperspektivische Narration.

\section{HYBRIDER TEXT}

Dass es Nathansen gelingt, solche Zwischenräume zu etablieren, hängt wesentlich mit der hybriden Verfasstheit seines Textes zusammen. Die Hybridität des Erzählens ermöglicht die Erkundung der Zwischenräume, und diese wiederum erfordern eine Textlichkeit, welche die Polyvalenzen zum Klingen bringen kann. Als hybrid lese ich dabei in Anlehnung an Elisabeth Bronfen „alles, was sich einer Vermischung von Traditionslinien oder von Signifikantenketten verdankt, was unterschiedliche Diskurse und Technologien verknüpft, was durch Techniken der collage, des sampling, des Bastelns zustandegekommen ist.“ (Bronfen, Marius, 1997: 14)

\footnotetext{
${ }^{6}$ Zitat im Original auf Deutsch.
} 
Nathansen bedient sich beispielsweise der Collagen-Technik, die unterschiedliche Sichtweisen gleichzeitig zulässt: Ständig wechselt die Erzählperspektive, der auktoriale Erzähler erweist sich zum einen als zutiefst unzuverlässig, zum anderen verschwindet er immer wieder. Dann lässt Nathansen die Perspektive des Protagonisten aber auch anderer Figuren nach vorn treten, die teils in sehr bruchstückhafter Weise ihre Gedankenströme teilen. Neben Briefe treten ausführliche Beschreibungen von räumlichen Anordnungen, offenen Fenstern und Türen, Düften und Licht-Schattierungen - Nathansens Vignetten lesen sich wie erzählte Hammershøj-Bilder: Durchgangsräume mit Aussicht. Immer wieder ,sampelt" Nathansen einzelne Phrasen, die refrainartig wiederholt den Roman rhythmisieren. Im Zentrum steht dabei die Gegenwärtigkeit des Erzählten bzw. Erlebten. Hugo David bewegt sich immer im absoluten Präsens, was im Umkehrschluss dazu führt, dass er keine durchgehend „schlüssigen“ Positionen bezieht, vielmehr sich ständig widerspricht: Das Theater ist der große Segen, die Rettung und die neue Familie. Kurz darauf schildert Hugo die Bühne als Unort, bevölkert von mittelmäßigen Mimen, deren Handeln ausschließlich darauf ziele, die Mitspieler lächerlich zu machen. Nicht anders ist es in der Liebe: Natürlich ist seine neue Bekannte Polly die allerschönste Frau, nie will er von ihr getrennt sein, heiße Gefühle in einem ewigen Frühlingsduft und Vogelgezwitscher.

„Nu kom den lykkelige Tid, da man slaar Øjene op med et Smil och lytter henrykt til Spurvekvidderet udenfor sit Vindue, mens et elsket Navn synger i Ens Øre. Nede fra Gaden lyder Fodtrin og Skramlen af Vogne, mindende et Øjeblik om Arbejdet, der kalder, og Hverdagens Pligter ... bort, bort i det Blaa ... Dagen er min ... Verden er min! Ungdomens Tid! Naar Hugo stod ved sit aabne Kvistvindue paa Nørregade og stirrede ud over Tagene, blev alting blaat for hans Øjne, blaat som Himlen, uendelig blaat som hendes fjærne Blik, der søgte mod hans gennem Luften - og han lukkede sine Øjne og syntes, at Luften var hendes Aande, der strejfede hans kind og Øjenlaag.“ (AHDL II: 65)

Aber schon spaziert Hugo in absoluter Ergriffenheit mit Ingeborg durch Kopenhagen, Liebesschwüre und Zukunftspläne werden geschmiedet und nur das Erzählwetter - immer strahlt die Frühlingssonne in einer von Veilchenduft geschwängerten Luft - ändert sich nicht. Wieder eine „Wendung“ und wir stehen mit Hugo in $\operatorname{Karls}^{7}$ Garderobe im Theater, ein duftender, nackter Traumkörper:

${ }^{7}$ Karl ist seit Kindertagen die große, lichterfüllte Sehnsucht Hugos, ein „kleiner Gott“: „Hugo saa', hvor hans [Karls] Bryst var stærk og hvalvet i Gangen, og Nakkens Linje skød sig rank og fast fra Kroppen - der laa som en Udfordring til Himlen i hans løftede Hoved med den lyse Lød, hvorover Solen gød sig med Velbehag. Og Hugo bøjede sit Hoved under denne Tryghed og Styrke, han følte sin Krop og sine Lemmer svagere en ellers.“ (AHDL II: 141) 
„Da han [Hugo] var færdig, gick han hen og bankede paa hos Karl. /.../ Karl sad nøgen i sin Badekaabe, Klæder og Linned flød over Stolen, han selv sad optaget foran Sminkespejlet, foer over Ansigtet med gult og hvidt, snakkede og lo op for sent paa Færde som sædvanlig, med Uret for sig som en Professor paa Kathedret. /.../ Han rejste sig, stod nøgen midt i Værelset, hans Krop var blevet noget sværere over Bryst og Lænd, men Benene var drengeagtig slanke - rank og ligevægtig stod han dèr som en ung Gud, straalende af Smidighed og Kraft. Hugo vendte sig bort, han følte en vis Sky for Karls hensynsløse Mandbarhed. Duften af den varme Hud blandede sig med Sminken og et lummert Pust af Parfyme. Stod han ikke dèr som et Billede af Teatret, en Krop til Beskuelse, en Prostitution af Kunstens Væsen...“ (AHDL III: 105f)

Ähnlich verhält es sich mit der Hingabe zur Schulfreundin Lis, der Jugendliebe Anna Blixen und dem Schauspieler Arthur Goldfeld. Der Roman liefert keine Erzählung über die eine große, sinnstiftende Liebe - vielmehr gibt es derer viele, immer absolut, voller Spannung und Leidenschaft. Keine weniger intensiv als die andere. Damit entsteht eine Gleichzeitigkeit von Möglichkeiten, die etliche Facetten des Romans prägt. Dieser Erzählmodus lässt sich vielleicht am ehesten als theatralisch bezeichnen, Nathansen erzählt über das Theater und wie das Theater: Das emotionale Jetzt, unruhig und unbeständig, bricht die bisweilen eintretende Schwere des Gesagten, weil absehbar ist, dass es sich immer nur um einen (vorübergehenden) Zustand handelt, keineswegs um abschließende Betrachtungen. Af Hugo Davids Liv zeichnet Aussagen, Einschätzungen und Beobachtungen als transitorisch, so nah dem Theatererlebnis wie möglich.

Wenn man diese spezifische Erzählweise Nathansens versteht und in Betracht zieht, lassen sich die Reflexionen darüber, wie ein „edler“ Jude zu sein habe, vielschichtiger betrachten, denn auch sie ergeben nur einen „Sinn“ im Gesamtklang des Geschriebenen, der Multiperspektivität der Standpunkte.

\section{8. „EDLE JUDEN“}

Auffällig ist, dass Nathansen in seinem Roman eine Vielzahl jüdischer Figuren auftreten lässt, die sehr verschieden erzählt werden und die sehr Verschiedenes erzählen - die immer wieder jedoch mit der Frage befasst scheinen, wie ein „edler und hilfreicher Jude“ in der (Kopenhagener) Gesellschaft zu agieren habe. Wir treffen dabei auf Charaktere unterschiedlicher Generationen, einige sind Teil von Hugos Familie, andere Freunde oder zufällige Bekannte, wieder andere bleiben Fremde. Nathansen mischt Stadt- und Landbewohner auch wenn ein großer Teil des Romans in Kopenhagen spielt - sowie Dänen, Italiener und Deutsche. Einige tauchen sporadisch auf, andere begleiten den Protagonisten bis zum Ende: Neben den Eltern und der Schwester Hugos begegnen wir seinen Tanten und Onkeln in Kopenhagen, treffen wir Cousinen 
und Cousins, seinen Jugendfreund, den ambitionierten Autoren Harry Hertz ein Begleiter durch das gesamte Buch, den deutschen Schauspieler Goldfeld (Hugo trifft ihn in Berlin), den Geschäftsmann Isidor, bei dem Hugo seine Ausbildung beginnt und den Kopenhagener Bonvivant Jacob Salomon, der gemeinsam mit seiner Frau einen der beliebtesten Salons der Hauptstadt führt. Es ließen sich noch etliche weitere Charaktere aufzählen, aber bereits dieser kurze Einblick verrät, wie bunt und vielstimmig sich das jüdische Leben in Nathansens Roman ausnimmt. Auf diese Weise weigert Nathansen sich, das jüdische Leben zu zeichnen - die Ambivalenz des Erzählten macht es schier unmöglich. Mit der Vielzahl der Figuren gehen unterschiedliche Standpunkte einher, wie ein ,edler Jude“ zu sein habe, was ihn auszeichne. Anders als es Poul Duedahl darstellt, fokussiert Nathansen dabei keineswegs auf einen „Rassenbegriff", der das Jüdische vermeintlich determiniert (Duedahl, 2017: 151). Dies ist eine Sicht, eine Möglichkeit, eine Stimme unter vielen. Aber sie wird ergänzt und, wie ich meine, deutlich hinterfragt, indem Nathasnen etliche weitere Positionen, Auffassungen und Argumente dazu stellt. Mit Blick auf die Vielstimmigkeit dieses großen Romanwerkes erscheint es mir zentral, dass Nathansen eben keine eindeutigen Antwort gibt, indem er viele Antworten gibt. Eine Essentialisierung „des Jüdischen“ wird damit obsolet. Gleichzeitig bleibt aber die Markierung besonderer Erfahrungen, die eben nicht im großen Karneval einer sich feiernden Hauptstadt spurlos verschmelzen.

Ich möchte dies an einem Beispiel ausführlicher diskutieren: Als Hugo beschließt, Schauspieler zu werden, löst dies geteilte Reaktionen aus. Nicht nur weil es sich um einen vermeintlich unsicheren Beruf handelt, sondern auch, weil er damit, so die Anmerkungen des Familienfreundes Jacob Salomon, das Jüdische in besonderer Weise sichtbar und damit angreifbar mache. In Jacobs Ausführungen, einer Art Gespräch mit Hugo, zeigt sich nun aber keine Stellungnahme des Autors, sondern eine von vielen Perspektiven, Auffassungen und Schattierungen:

„Ja!“ sagde han [Jacob Salomon] saa med et pludseligt Sæt, idet han vendte sig i Stolen, ,jeg ønsker at tale et Øjeblik med Dem, hvis De tillader - Tak - ganske privat - Altsaa: Jeg hører af Far, at De omgaas med Planer om at gaa til Scenen det er en meget uheldig Omgang, synes jeg, ja undskyld, jeg taler lige ud af Posen - meget uheldig! Naa, De har truffet Deres Bestemmelse - jeg forstod paa Far i Telefonen, at Spørgsmaalet endnu stod aabent $-/ . . /$ men De har maaske ikke noget imod at høre et Par Ord fra et Menneske, der kender lidt til Livet bag Kulisserne og, ved Gud, mener Dem det godt. - Glæder mig! Altsaa: Hvad vil De dèr? - Selvfølgelig, selvfølgelig - det vil vi gærne alle - efter ringe Evne - men vi har unge Jøder nok, der søger at paatvinge sig Offentlighedens Opmærksomhed og skader os andre med deres Jødedom og Chrutspe. Hvadbehar - kommer det ikke Sagen ved? Tillader De? Tak. Jeg mener kort og godt, at vi bør holde os indenfor vort Omraade - Forretning, ja - Videnskap, gudbevares - men Kunst! Tror De 
maaske, Goldschmidt har gavnet os ... jeg finder ham væmmelig ... al den Mouschlen og sentimentale Snak om Hjemløshed ... hvad maa man tro om os? Indblik - ja, Godmorgen - - og Forstaaelse siger De! Hvad kommer det mig ved? /.../ Gudbevares - for min Skyld - hvis De absolut er uimodtagelig for Fornuft ! Men jeg siger Dem, det er en Sækkegade, min unge Herre - De vil blive ildeset af de andre og mistænkeliggjort af Deres egne!“” (AHDL II: 177ff)

Jacob Salomon plädiert hier recht eindeutig für eine „Unsichtbarmachung" des Jüdischen, mit anderen Worten für eine weitreichende Assimilation. An anderer Stelle illustriert er dies, indem er bekennt, dass er seine Kinder am liebsten taufen ließe und lediglich aus Rücksicht auf seine Eltern davon Abstand nehme. Vor diesem Hintergrund verwundert es nicht, dass Jacob die Bühnenkarriere des Protagonisten zu verhindern sucht, da diese gerade auf eine positive Sichtbarmachung ausgerichtet ist. Interessant erscheint mir hierbei, dass die unterschiedlichen Standpunkte mit einer Generationenfrage verknüpft werden: Hugo und die anderen ,jungen Juden“ torpedieren die Assimilationsbestrebungen Jacobs und damit die Assimilationsbestrebungen einer ganzen Generation. Dass dies als Konflikt zwischen Jung und Alt erzählt wird, löst die Auseinandersetzung zumindest teilweise aus einem rein jüdischen Spektrum und illustriert die beständigen intergenerationellen Aushandlungsprozesse zwischen Veränderung und Beharrung - die natürlich auch und vielleicht in besonderer Weise die jüdische Gemeinde Kopenhagens seit dem Ende des 18. Jahrhunderts treffen (Räthel, 2016: 124), aber verdeutlichen, dass der Wunsch nach Sichtbarmachung bzw. Unsichtbarmachung beständig Konjunkturen unterliegt.

Kennzeichnend ist, dass Hugos Standpunkt in dem Gespräch nur indirekt vermittelt wird. Hier tritt Nathansens augenfällige Verwendung des Dialogs zu Tage. Weite Teile der vier Bände bestehen - ähnlich wie der gerade zitierte Ausschnitt - aus dialogartigen Versatzstücken, die sich dadurch auszeichnen, dass die Redeanteile nur eines der Gesprächspartner wiedergegeben werden, in der Regel nicht Hugos Anteile am Gesagten. Mit Hilfe dieser Form - ich wähle die Bezeichnung Mono-Dialog - liefert Nathansen eine Vielzahl von Puzzleteilen, die kein fertiges Ganzes ergeben, sondern immer wieder neu arrangiert, unterschiedliche Perspektiven zulassen, die weder bewertet noch hierarchisiert werden, sondern gleichwertig nebeneinander stehen können.

Nathansen überlässt es aber nicht allein seinen jüdischen Charakteren, die Ausgestaltung jüdischen Lebens in Dänemark zu diskutieren. Er lässt auch nicht-jüdische Figuren dieser Frage nachgehen. Ein prominentes Beispiel zeigt das Gespräch zwischen den beiden Malern Vilhelm Holst und Lars Fjordby im zweiten Band, die in Hugos Anwesenheit stark widersprüchliche Positionen zur „Judenfrage“ einnehmen. Holst erklärt eindringlich, er liebe die Juden (AHDL II: 46) und preist ihren Beitrag zur kulturellen Entwicklung des Landes; Fjordby hingegen steht auf dem Standpunkt, dass sich die Kultur nie ge- 
gen die jüdische Natur durchsetzen könne, die er als degeneriert ansieht. Die vermeintlich einfache Lesart, Fjordbys Positionen als judenfeindlich abzulehnen, wird dadurch erschwert, dass Hugo und er sehr herzlich miteinander umgehen, Hugo nach der Diskussion Partei für ihn ergreift und Gemeinsamkeiten ausmacht: ,[J]eg føler mig paa en vis Maade beslægtet med Fjordby han hører jo selv til et Lag, der har været holdt i Skyggen gennem Aarhundreder og nu fordrer sin Part af Livet og Lyset.“ (AFHDL II: 52) Fjordbys Außenseiterposition - er stammt aus der bäuerlich geprägten Provinz und hat sich als Künstler trotz dieser Voraussetzungen behaupten können - zeigt Schnittmengen mit Hugos. Fjordbys Idee vom Judentum zielen auf das großstädtische Milieu, das ihn aus seiner Sicht hart und unwillkommen als den bildungsfernen Neuankömmling belächelt.

Diese Mehrfachbelichtung von Migrationserfahrung und Zwischenpositionen zieht den Blick von einer einseitig jüdischen Erfahrung zu einer universelleren Zeichnung: Das Gefühl, außen zu stehen, wird nicht als eine exklusiv jüdische Erfahrung bewertet. Das Theater, über weite Strecken Hugos Zuhause, ist bevölkert von Menschen, die sich dazwischen fühlen. Auch außerhalb der Bühne portraitiert Nathansen etliche Außenseiter: Wirtschaftlich abgehängt findet Hugos ehemaliger Schulfreund Aage Miller nach dem Bankrott nicht mehr zurück in die Gesellschaft; die gesellschaftlich gut gestellte Frau Bang liebt unglücklich - trotz ihres Wohlstandes bleibt sie eine beständig Irrende. So findet sich im gesamten Roman eigentlich keine Figur, die dazugehört, die ein Ziel gefunden hat. Fast scheint es, in ganz Dänemark gäbe es keine verbindenden Wege oder Brücken, keine Aussicht auf ein An- und Zusammenkommen: „Der er for mange Øer i Danmark - tror De ikke? Vi sidder hver paa sin $\varnothing$, adskilt ved Bælter, og Sunde, hver med sit Hjemsted, sin Natur, sit individuelle Væsen." (AHDL III: 277) So lässt sich Af Hugo Davids Liv auch als Inselerzählung lesen. Die mehrfachbelichtete Personengalerie sowie die Mehrstimmigkeit des hybriden Textes lassen dabei einen literarischen Raum entstehen, der, wie Søren Frank fordert, kulturelle und nationale Identitätsvorstellungen zentrifugiert und monokulturelle Bilder von Nation und deren Bevölkerung mit weniger eindeutigen Bildern überschreibt. (Frank, 2012: 6)

\section{WER SPRICHT?}

Ich möchte noch einmal auf die oben zitierte „Unterhaltung“ zwischen Jacob und Hugo zurückkommen und auf einen weiteren wichtigen Gesprächsgegenstand hinweisen: Das Verhältnis der beiden Figuren zu Meïr Aaron Goldschmidt. Goldschmidts vielfältiges literarisches und publizistisches Wirken fokussiert - grob vereinfacht - auf die jüdische Erfahrung in Dänemark zur Mitte des 19. Jahrhunderts. Besondere Relevanz entwickeln dabei seine Romane Hjemløs (erschienen zwischen 1853 und 1857) sowie En Jøde aus dem 
Jahr 1845, auf letzteren bezieht sich Af Hugo Davids Liv in vielen Abschnitten direkt oder indirekt.

Jacob verwehrt sich entschieden gegen die Goldschmidt'sche Sicht auf das Jüdische, die er als selbstmitleidige Anklage gegen Diskriminierung liest. Hugo hingegen nimmt Aspekte auf und inkludiert sie in seine Überlegungen, gleichzeitig klingt in Hugos Verhältnis zu Goldschmidt das Selbstverständnis einer jungen Generation an, die Dänemark als ihr Land betrachtet und sich weniger fremd fühlt oder fühlen will. Der Protagonist verweigert sich Goldschmidts Idee der klaren Abtrennung - man könne entweder Jude oder Däne sein: „Jeg husker, hvad jeg følte, da jeg læste Goldschmidts Ord: Jeg er en Jøde, hvad vil jeg iblandt jer? /.../ Jeg forstaar ham, men saadan føler jeg det ikke." (AHDL II: 52) Vielmehr ersetzt er das Narrativ durch den Befund, dass das Dazwischenstehen - oder eben auch die Gleichzeitigkeit auf beiden Seiten platziert zu sein - kennzeichnend und möglich sein könne:

„Jeg staar jo paa en mærkelig Maade udenfor /.../: Landet er mit - ogsaa jeg har Ret til at være her! Men samtidig véd jeg ogsaa, at jeg aldrig faar Ret, /.../jeg véd, at jeg maa bevise min Ret, og det kan jeg ikke, eller maaske: det vil jeg ikke. For jeg kunde ikke gøre det uden at svigte noget dybt rodfæstet i min Natur, der kræver Del i de andres uden dog at kunne slippe sit eget, længes bort fra mig selv og længes atter tilbage, naar jeg er borte.“ (AHDL II: 52)

Af Hugo Davids Liv gebärdet sich dabei keineswegs als Abrechnung mit Goldschmidt, wie beispielsweise Tine Bach analysiert. (Bach, 2004: 232) Vielmehr dient das literarische Vorbild als wichtiger Referenzrahmen. Die vielgestaltige Auseinandersetzung mit dem Autor Goldschmidt und seinem Werk - allen voran mit dem Entwicklungsroman En Jøde - verstärkt dabei die Mehrstimmigkeit jüdischer Erfahrungen und Positionen in Nathansens Roman.

Mit Blick auf die ständig auftauchenden Auseinandersetzungen mit Goldschmidt entwickeln zudem Goethe und die Goethe-Rezeption in Af Hugo Davids Liv eine besondere Relevanz. Wie eingangs beschrieben, steht der an Goethe angelehnte Wunsch, als Jude edel, hilfreich und gut zu agieren, wie eine Überschrift über dem Roman. Damit zielt Nathansen nicht nur auf das Gedicht Das Göttliche, sondern gleichzeitig auch auf den heute vergessenen, damals jedoch äußerst erfolgreichen Roman von Ludwig Jacobowski Werther, der Jude aus dem Jahr 1892. Es ist dessen Protagonist, Leo Wolf, der diesen Vorsatz fasst und auf den Hugo David referiert. Und Leo Wolf beschäftigen ganz ähnliche Fragen, wenn auch in einem gänzlich anderen Milieu, mit anderen Herausforderungen und Konsequenzen.

Auch bei Jacobowski treffen wir auf einen jungen Juden, der seinen Platz in der Gesellschaft sucht. Leos Wunsch nach Teilhabe wird jedoch als Werther-Faust-Verschnitt präsentiert: Es gibt eine - natürlich - blonde Helene, die von Leo ein Kind erwartet, während dieser sich zu einer verheirateten Frau 
hingezogen fühlt. Helene bringt sich aus Verzweiflung und Scham um - immerhin ist es nicht nur ein uneheliches, sondern auch ein jüdisches Kind, das sie erwartet -, worauf sich Leo in Werther'scher Manier erschießt. Jacobowski zeigt - in der Handlung aber auch im narrativen Gestus - wie sein Protagonist bis in den ästhetisierten Freitod hinein versucht, die deutsche Mehrheitsgesellschaft und deren kulturelle Codes zu imitieren. (Anderson, 1996: 201) Dies illustriere, so liest es Anderson, dass bei Jacobowski Assimilation eben keine „Symbiose von Identitäten“ produziere (Anderson, 1996: 204), sondern dem Protagonisten nur ein unbehauster Platz im Dazwischen zugewiesen werde.

Im Dreiklang zwischen Goldschmidts En Jøde, Jacobowskis jüdischem Werther und Nathansens Roman tritt das Besondere an Af Hugo Davids Liv hervor. Nathansen schreibt keine Assimilationsphantasie, wie sie bei Jacobowskis Werther durchscheint (Hess, 2005: 210), aber er folgt ebenso wenig Goldschmidts Spur der Integrationsunmöglichkeit. Vielmehr bietet er einen im besten Sinne ziellosen Roman, der Migration nicht als den Weg von einem zum anderen Ort erzählt und damit Integration ebenfalls nicht als Auflösung von Gegensätzen. Die bei Jacobowski fatalistisch aufgeladenen Zwischenräume öffnet Nathansen als produktive Möglichkeitsorte, zeigt sie in ihrer hybriden Verfasstheit und vielstimmigen Figuration. In Nathansens Niemandsländern wimmelt es von Migranten im Søren'schen Verständnis: nichtangekommenen Figuren und Konzepten.

Neben diese Goldschmidt- und Jacobowski-Bilder bastelt Nathansen noch weitere stimmgewaltige literarische Vorgänger in seinen Roman hinein: Collagenartig zusammengesetzt werden Werkzitate von Friedrich Schiller, Thomas Mann, Ludvig Holberg, Karl Gutzkow, William Shakespeare, Johann Hermann Wessel, Nathansens eigenem Schaffen ${ }^{8}$ und immer wieder Lieder und Gedichte aus Heinrich Heines umfangreichem Oeuvre. Oft wird nicht klar, wer eigentlich gerade spricht: Nathansen, Hugo, weitere Figuren, „fremde“ Autoren, deren Protagonisten? Dieses intertextuelle Hintergrundrauschen unterstreicht zum einen die Hybridität des Textes, zum anderen aber erzählt es Zwischenpositionen multiperspektivisch - und erneut nicht exklusiv jüdisch: Wenn beispielswiese Thomas Manns Tonio Kröger zu Wort kommt und verlautet: ,[E]tliche gehen mit Notwendigkeit in die Irre, weil es einen rechten Weg für sie überhaupt nicht giebt. /.../ Ich stehe zwischen zwei Welten, bin in

\footnotetext{
${ }^{8}$ Im dritten Teil spielt Nathansen beispielsweise auf seine eigene literarische Tätigkeit an, indem er Harry im Roman ein Stück schreiben lässt, das augenfällig an sein 1915 erschienenes Drama Dr. Wahl erinnert. Die Aufführung - mit Hugo und Karl in den Titelrollen - misslingt. Hugos Theaterlehrer Professor Rosen analysiert danach raffiniert, woran die Aufführung gescheitert ist: Sie wollte zu offensichtlich belehren. Bedenkt man diese Konstellation, dann scheint auch hier die Hybridität des Textes auf: Nathansen schreibt und inszeniert ein Stück, das nur bedingt erfolgreich war (Räthel, 2018b), kritisiert und analysiert die Aufführung dann kurz darauf selbst in seinem Roman.
} 
keiner daheim und habe es infolge dessen ein wenig schwer." ${ }^{\text {"9 }}$ (AHDL IV: 41), dann bleibt zunächst in der Schwebe, wer eigentlich spricht - bevor Nathansen die Bewohner seiner Dritten Räume vorstellt: Hugo sitzt zusammen mit seinem alten Schulkameraden, dem ,roten Johannes“, einem verwitweten Außenseiter mit Kind, kaum akzeptiert in der Gesellschaft, der Anschluss vor allem in seiner Bibliothek mit „hjemlig og fremmed Litteratur“ (AHDL IV: 37) findet.

Der Text offeriert damit durchgängig zeitliche und räumliche Überlagerungen von Zwischenpositionen, die gleichberechtigt miteinander kommunizieren. Liest man Af Hugo Davids Liv auf diese Weise, scheint es nicht, als würde Nathansen die Migrationserfahrungen zu Beginn des Jahrhunderts und die daraus erwachsenden gesellschaftlichen Spannungen ignorieren. Vielmehr macht er die Kakophonie seiner vielschichtigen Erzählung hörbar, die Migration als immerwährend zeichnet.

\section{EROTIK DES DRITTEN RAUMES}

Der Versuch, die Vielstimmigkeit des Romans herauszustellen und die produktiven Möglichkeiten der Zwischenräume in Af Hugo Davids Liv zu beleuchten, soll nicht verbergen, dass Nathansen ein spannungsgeladenes Portrait seiner Zeit zeichnet - keinen paradiesischen Lustgarten -, das immer wieder überrascht, inkonsistente Brüche besichtigt und komplexen und komplizierten Formationen Raum gibt. Die Frage, wie ein edler, hilfreicher und guter Jude zu sein habe, bleibt dabei offen. Aber die Tatsache, dass die Frage als notwendig formuliert wird, verrät viel über die zeitweilige Unruhe der Figuren. In diesem aus einem ständigen Bekenntnisdruck erwachsenen Unbehagen treffen sich exemplarisch Hugo und sein Schulfreund und Lebensbegleiter Harry Hertz auf einem Spaziergang. Zwei junge dänische Juden - einsam, aber nicht allein beichten bei Nacht:

„Nu som idag for Eksempel - hvad Indtrykk har du haft af de andre - synes du, du hører dem til? Ja, ja, men kun til et Punkt - Ligheden hører op, hvor det ny Testamente begynder. /.../ [H]er, hvor vi er alene og begge Jøder for Vorherre og ikke engang kan se hinanden rødme, her kan vi aabent tale ud om Sagerne. Hvis vi ikke tilfældig var Jøder, vilde vi maaske hade hinanden, nu føler vi os forbundne under den samme ulykkelige Stjærne.“/.../

[Hugo rakte] sin Haand ud mod Harry, der stod nogle Skridt fra ham med løftet Hoved i Natten som dengang - og sagde: „Jeg ved ikke, Harry - det er, som om vi aldrig faar Ro. Er det en daarlig Samvittighed overfor os selv, fornægter og bekræfter vi hinandens Natur, samtidig med at vi fornægter og bekræfter de andres? /.../ Jeg er Jøde som du, Harry - nu da vi staar her med hinanden i Haanden og ik-

\footnotetext{
${ }^{9}$ Zitat im Original auf Deutsch.
} 
ke kan se hinanden rødme, som du siger, kan jeg sige dig det. /.../ Dèr ligger Livet, Harry .. for $o s--$ Drømmen om Grevskabet østen for Sol og vesten for Maane - som vi aldrig skal naa." (AHDL II: 152ff)

Und so erwächst aus dem nächtlichen Versteckspiel ein weiterer, märchenhafter Sehnsuchtsort, welcher (unerreichbar) östlich der Sonne und westlich des Mondes platziert wird. Jüdischsein produziert nicht zwangsläufig Gemeinsamkeiten, vielmehr schimmert auf, als wie vielfältig aufgeladen sich die Nathansen'schen Zwischenräume gerieren, behaust von unsicheren, errötenden Charakteren, die sich in spannungsgeladenen Aushandlungsprozessen immer wieder refigurieren.

Dèr sad de [Harry og Hugo] Side om Side, hinanden nær og dog saa forunderlig fjærn - var det den fælles Nød i deres Hjærter, den haarde Skal af Mistænksomhed og Selviskhed, der lukkede for en Hengivelse, som fortærede sig selv, fordi dens Glød ikke kunde finde Vej til et andet Hjerte? Hvorfor gav de ikke efter for deres hemmelige Trang, hvorfor lod de ikke det forpinte Hjærte strømme ud, varmt og fuldt af Glæde over at give sig hen? /.../ Havde de mange Aarhundreders Utryghed og Angst skabt et Bundfald af Fejghed i deres Væsen, der angstedes for, at den glødende Trang til Hengivelse skulde røbe deres inderste Hemmelighed, hvis den fik Lov til aabent og uhindret at strømme du - skulde blotte deres dybeste Natur i dens kvindelige sarte Følsomhed, i dens blege Svaghed, der søgte Støtte og Hvile efter Aarhundreders ensomme Kamp? (AHDL II: 210f)

Nathansen zeichnet seine Dritten Räume vielgestaltig, ihre auch erotische Aufladung lässt sich kaum negieren. Ich meine, dies hat nicht nur mit dem Verhältnis zwischen Harry und Hugo zu tun, sondern es unterstreicht auch die Anziehungskraft des Dritten Raums. Nathansen zeichnet diesen Ort dabei nicht als defizitären Spielraum, aus dem der Protagonist - und alle seine MitbewohnerInnen - manchmal mehr, manchmal weniger erfolgreich zu fliehen suchen, er erzählt damit ebenfalls keine jüdische Heimatlosigkeit. Vielmehr ermöglicht er, den Zwischenraum als unbequeme, sinnliche, vor allem eigenständige Möglichkeit der Existenz wahrzunehmen. Wenn wir also diese virulenten Zwischenpositionen als narrativen ,third space“ lesen, ihn im Sinne Homi Bhabhas zentrifugieren, kann es gelingen, Nathansens Roman weder als Wunsch und Bemühen nach Assimilation zu deuten noch deren Unerreichbarkeit zu zelebrieren, sondern vielmehr den Blick darauf zu richten, dass er Ränder, Grenz- und Überlappungszonen produktiv gestaltet (Bachmann-Medick, 2009: 197f.) und die (Un-)Möglichkeiten von Fremdheit schmerz- und lustvoll beleuchtet. 


\section{LITERATURVERZEICHNIS}

Anderson, M. (1996). Jewish Mimesis? Imitation and Assimilation in Thomas Mann's Wälsungenblut and Ludwig Jacobowski's Werther, der Jude. German Life and Letters 49 (2), S. 193-204.

Bach, T. (2004). Exodus. Om den hjemløse erfaring i jødisk litteratur. Hellerup: Spring.

Bachmann-Medick, D. (2009) Cultural Turns. Neuorientierungen in den Kulturwissenschaften. Reinbek bei Hamburg: Rowohlt.

Benjamin, W. (1989). Was ist das epische Theater? (1) Eine Studie zu Brecht. In: Walter Benjamin: Gesammelte Schriften II, 2, herausgegeben von Rolf Tiedemann und Hermann Schweppenhäuser (S. 519-531). Frankfurt am Main: Suhrkamp.

Bhabha, H. (2000). Die Verortung der Kultur. Tübingen: Stauffenburg-Verlag.

Blum, J. (1973). Dansk og/eller jøde? En kultursociologisk undersøgelse af den jødiske minoritet $i$ Danmark. Kopenhagen: Gyldendals Samfundsbibliotek.

Borchsenius, P. (1969). Historien om de danske jøder. København: Fremad.

Bronfen, E., Marius. B. (1997). Hybride Kulturen. Einleitung zur anglo-amerikanischen Multikulturalismusdebatte. In: Bronfen, E., Marius. B. (eds.), Hybride Kulturen (S. 1-29). Tübingen: Stauffenburg-Verlag.

Duedahl, P. (2017). Fra overmenneske til UNESCO-menneske. Racebegrebet i Danmark 18901965. Odense: Syddansk Universitetsforlag.

Frank, S. (2012). Hvad er migrationslitteratur? Kritik Nr. 203, S. 2-10.

Jørgensen, N.P. (1999): The Stage Jew. In: Gelfer-Jørgensen, M. (ed.), Danish Jewish Art. Jews in Danish Art (S. 470-479). København: Rhodos.

Leicht, G. \& Hallar, M. (1977). Det kongelige Teaters repertoire 1889-1975. Ballerup: Bibliothekscentralens Forlag.

Müller-Wille, K. (2013). Ende gut, alles gut? Das Imaginäre der Ökonomie und die Konstitution des Populärtheaters (Fasting, P.A. Heiberg, Overskou, Hertz). In: Müller-Wille, K., Schiedermair, J. (eds.), Wechselkurse des Vertrauens. Zur Konzeptualisierung von Ökonomie und Vertrauen im nordischen Idealismus (S. 193-213). Tübingen: Francke.

Nathansen, H. (1917). Af Hugo Davids Liv. 4 Bd. København: Pios Boghandel.

Hess, J. (2005). Fiction of a German-Jewish Public: Ludvig Jacobowski's Werther the Jew and Its Readers. Jewish Social Studies 11 (2), S. 202-230.

Kulturministeriet (ed.) 2006 Kulturkanon. København: Politikens Forlag.

Räthel, C. (2016). Wie viel Bart darf sein? Jüdische Figuren im skandinavischen Theater. Tübingen: Narr/Francke/attempto.

Räthel, C. (2018a). What's Jewish about a Jew? The Question of (Un-)Recognizability in Two Productions of Henri Nathansen's Play Indenfor Murene (Within the Walls). Scandinavian Studies 90 (1), S. 23-49.

Räthel, C. (2018b). Could You Change the Final Act? Prosesses of Translation in and around Henri Nathansen's Play Dr. Wahl. In: Berni, B., Wegener, A (eds.): Translating Scandinavia: Scandinavian Literature in Italian and German Translation, 1918-1945. Rom: Edizioni Quasar, S. 141-152.

Schiedermair, J. (2013). Der Kaufmann von Kopenhagen. Geld und Gabe in Thomasine Gyllembourgs Novelle Jøden (1836) In: Müller-Wille, K., Schiedermair, J. (eds.), Wechselkurse des Vertrauens. Zur Konzeptualisierung von Ökonomie und Vertrauen im nordischen Idealismus (S. 51-68). Tübingen: Francke.

Schnurbein, S. (2004). Darstellungen von Juden in der dänischen Erzählliteratur des poetischen Realismus. In: Nordisk Judaistik. Scandinavian Jewish Studies 25 (1), S. 57-78.

Schnurbein, S. (2007). Hybride Alteritäten. Jüdische Figuren bei H.C. Andersen. In: Behschnitt, W., Herrmann, E. (eds.), Über Grenzen. Grenzgänge der Skandinavistik. Festschrift zum 65. Geburtstag von Heinrich (S. 129-150). Würzburg: Ergon-Verlag. 
Rutherford, J. (1990). The Third Space. Interview with Homi Bhabha. In: Rutherford. J (ed.), Identity. Community, Culture, Difference (S. 207-211). London: Lawrence \& Wishart.

Svenson, A. (2016). Om Henri Nathansens flyktingtid och död. Några anteckningar. Rambam. Tidsskrift for jødisk kultur og forskning (25), S. 140-148.

\section{Clemens Räthel}

Humboldt-Universität zu Berlin

Nordeuropa-Institut

Unter den Linden 6

10099 Berlin

Deutschland

clemens.raethel@hu-berlin.de 